\title{
Structural Implications of Mutations in the Pea SYM8 Symbiosis Gene, the DMI1 Ortholog, Encoding a Predicted Ion Channel
}

\author{
Anne Edwards, ${ }^{1}$ Anne B. Heckmann, ${ }^{1}$ Faridoon Yousafzai, ${ }^{2}$ Gerard Duc, ${ }^{3}$ and J. Allan Downie ${ }^{1}$ \\ ${ }^{1}$ Department of Molecular Microbiology and ${ }^{2}$ Computational Biology Unit, John Innes Centre; Colney Lane, NR4 7UH, \\ Norwich, UK.; ${ }^{3}$ nstitut National de la Recherche Agronomique, INRA-UR LEG, BP 86510, 21065 Dijon cédex, France
}

Submitted 10 May 2007. Accepted 15 June 2007.

\begin{abstract}
The Pisum sativum SYM8 gene plays an essential part in both rhizobial and mycorrhizal symbioses. Mutation of syms in the original type line R25 blocks nodulation, mycorrhization, and Nod-factor-induced calcium spiking, an early component of the nodulation signaling pathway. We describe four new sym 8 alleles of pea, which fall into the same complementation group as $\mathrm{R25}$. The sym 8 mutants are phenotypically similar to Medicago truncatula dmil mutants and map to a syntenic location. We used sequence homology to isolate the pea ortholog of M. truncatula DMII and have shown that the cloned pea ortholog can complement a $M$. truncatula dmil mutant for nodulation. Each of the five pea sym8 mutants carries a mutation in the DMII ortholog, confirming that the pea SYM8 is the DMII ortholog. Based on predicted structural similarities with an archaebacterial ion channel, we propose that SYM8 forms a tetrameric calcium-gated channel of a predicted structure similar to the archaebacterial potassium channel but containing a filter region that is different. The predicted structure identifies four aspartate residues (one from each subunit) forming the channel opening. We made a mutation changing the aspartate to valine and identified a missense mutation (changing alanine to valine adjacent to the aspartate residues) in this predicted filter region; both mutations caused a loss of function. We also identified a lossof-function missense mutation (changing arginine to isoleucine) in a domain proposed to link the predicted channel and the gating ring domains, indicating that this mutation may block function by preventing a protein conformational change being transmitted from the gating-ring domain to the pore domain.
\end{abstract}

Many leguminous plants can acquire two essential nutrients through symbioses. Phosphate can be acquired via arbuscular mycorrhizal fungi (Harrison 1999) and ammonium can be obtained from nitrogen-fixing Rhizobium bacteria. The establish-

Corresponding author: Anne Edwards; Telephone: +44 (0)1603 450172; Fax: +44 (0) 1603 450045; E-mail: anne.edwards@bbsrc.ac.uk

Nucleotide sequence data reported are available in the EMBL and GenBank nucleotide sequence databases under the accession numbers: EF447276, Sparkle SYM8 cDNA; EF447277, Finale SYM8 cDNA; EF447278, partial SYM8 genomic sequence from Finale; EF447279, sym8-1; EF447280, sym8-2; EF447281, sym8-3; EF447282, sym8-4; and EF447283, sym $8-5$.

*The $\boldsymbol{e}$-Xtra logo stands for "electronic extra" and indicates that Figures 2 and 4 appear in color online. ment of both symbioses depends on a signal transduction pathway, some steps of which are common to both fungal and bacterial interactions (Kistner and Parniske 2002). Of the two, the rhizobial symbiosis is better understood. Rhizobial Nod factors activate a signaling pathway in legume roots and this culminates in nodule organogenesis (Oldroyd and Downie 2004). $\mathrm{Ca}^{2+}$ spiking, which results from Nod-factor-induced oscillations in root-hair intracellular calcium in different legumes (Cardenas et al. 1998; Ehrhardt et al. 1996; Harris et al. 2003; Wais et al. 2000; Walker et al. 2000), is linked to the induction of early nodulation genes such as ENOD11. This requires the activity of a calcium-calmodulin-dependent kinase (CCaMK) (Levy et al. 2004; Mitra et al. 2004).

Different receptor-like kinases have been implicated in the perception of Nod factors. In Lotus japonicus, NFRI and NFR5 encode two LysM-type serine/threonine receptor kinases, which are thought to be involved in Nod factor binding (Madsen et al. 2003; Radutoiu et al. 2003). L. japonicus NFR5 is orthologous to Medicago truncatula NFP (Arrighi et al. 2006) whereas L. japonicus NFRl has two potential orthologs in M. truncatula, LYK3 and LYK4 (Limpens et al. 2003). Downstream of Nod-factor perception is a receptor-like kinase encoded in M. truncatula by DMI2 (Endre et al. 2002) and in L. japonicus by SYMRK (Stracke et al. 2002). Unlike the Nod-factor receptors, this gene product lies on the common symbiotic pathway required for both mycorrhization and nodulation (Stracke et al. 2002; Wais et al. 2000). Also on the common pathway are genes encoding integral membrane proteins, which may act as multimeric ion channels. Thus, in L. japonicus, there are two similar genes, CASTOR and POLLUX, both predicted to encode an ion channel; however, unexpectedly, these proteins were reported to be plastid-localized in $L$. japonicus (Imaizumi-Anraku et al. 2005) based on localization of green fluorescent protein (GFP) fusions. In M. truncatula, only one such symbiosis gene, DMII, appears to be present (Ané et al. 2004). DMI1 is more similar to POLLUX, but DMI1-GFP fusions were localized around the nucleus (Riely et al. 2007). Two additional genes whose products function between Nod-factor perception and $\mathrm{Ca}^{2+}$ spiking have been identified in L. japonicus; both are predicted to be nucleoporins (NUP133 and NUP85) and both also are required for mycorrhization and (Kanamori et al. 2006; Saito et al. 2007).

Knowledge gained from these model legumes has been exploited for syntenic studies on pea, for which there are many well-characterized nodulation mutants (Guinel and Geil 2002). Of the mutations known to lie upstream of $\mathrm{Ca}^{2+}$ spiking, Pisum sativum SYM10, predicted to be involved in Nod factor perception (Walker et al. 2000), is the ortholog of L. japonicus NFR5 
(Radutoiu et al. 2003); P. sativum SYM19 (Schneider et al. 1999) is orthologous to L. japonicus SYMRK (Stracke et al. 2002); and P. sativum SYM2 (Geurts et al. 1997) is thought to be the ortholog of M. truncatula LYK3 or LYK4 (Limpens et al. 2003). Thus far, the $P$. sativum SYM 8 gene, which is required for nodulation, mycorrhization (Balaji et al. 1994; Kneen et al. 1994), and calcium spiking (Walker et al. 2000), has not been characterized. $P$. sativum sym 8 mutants are phenotypically similar to M. truncatula dmil mutants (Walker et al. 2000) and the SYM8 and DMII genes map to a syntenic location in the two legumes (Ané et al. 2002), indicating that SYM8 is likely to be an ortholog of DMI1. This work describes four more sym 8 alleles. We isolated the pea ortholog of M. truncatula DMII and showed that the five sym 8 pea mutants carry mutations in this gene. Based on predicted structural similarities with the MthK channel from Methanobacterium thermoautotrophicum, we propose that SYM8 forms a tetrameric calciumgated cation channel and define key amino acids likely to be involved in calcium activation and cation selectivity.

\section{RESULTS}

Identification of five independent sym8 mutants.

Mutagenesis using ethyl methanesulphonate (EMS) generated a collection of $P$. sativum var. Finale mutants with altered nodulation phenotypes (Duc and Messager 1989; Engvild 1987). A subset of 37 mutants in the M3 and M4 generation lacked nodules and 20 of these mutants failed to establish a vesiculararbuscular mycorrrhizal symbiosis (Duc et al. 1989). Of these 20 mutants, genetic crosses revealed that four, RISNOD10, RISNOD19, RISNOD21, and RISNOD25, belong to one complementation group (Table 1). Back-crosses (to Frisson) with these mutants confirmed that the mutations are all recessive and the mutant phenotypes segregated $3: 1$, indicating that each mutant carries a single mutation affecting nodulation (Table 2). Some of these mutants were crossed with representatives of the sym8, sym19, and sym30 (allelic with sym9) $\mathrm{Nod}^{-} \mathrm{Myc}^{-}$mutant complementation groups (Table 1). This revealed that RISNOD21 carries a mutant allele of sym8 and it follows that RISNOD10, RISNOD19, and RISNOD25 carry mutant alleles of sym8. This complementation group is distinct from sym30 and sym19 groups (Table 1), which also are $\mathrm{Nod}^{-} \mathrm{Myc}^{-}$. The five mutant lines in the sym 8 complementation group were designated with

Table 1. Allelism test between mutants and against sym 30 , sym19, and sym 8 groups $^{\mathrm{a}}$

\begin{tabular}{lcccc}
\hline & RISNOD10 & RISNOD21 & RISNOD19 & RISNOD25 \\
\hline RISNOD10 & $-(30)$ & $\mathrm{NT}$ & $\mathrm{NT}$ & $\mathrm{NT}$ \\
RISNOD21 & $-(28)$ & $-(30)$ & $\mathrm{NT}$ & $\mathrm{NT}$ \\
RISNOD19 & $-(8)$ & $-(16)$ & $-(30)$ & $\mathrm{NT}$ \\
RISNOD25 & $-(2)$ & $-(5)$ & $-(8)$ & $-(30)$ \\
sym30 (P2) & $+(1)$ & $+(27)$ & $+(11)$ & NT \\
sym19 (P4) & $+(1)$ & $+(16)$ & $+(9)$ & $+(1)$ \\
sym8 (R25) & $\mathrm{NT}$ & $-(7)$ & $\mathrm{NT}$ & $\mathrm{NT}$ \\
\hline
\end{tabular}

${ }^{\text {a }}$ Symbols: + = wild phenotype and $-=$ mutant phenotype (number of F1 plants); NT = not tested.

Table 2. Inheritance of mutant phenotype in $\mathrm{F} 2$ progeny from sym 8 backcrosses with cv. Frisson

\begin{tabular}{lccccccc}
\hline \multirow{2}{*}{$\begin{array}{l}\text { Parental } \\
\text { lines }\end{array}$} & \multirow{2}{*}{$\begin{array}{c}\text { No. } \\
\text { analyzed }\end{array}$} & \multicolumn{2}{c}{ No. of plants } & & \multicolumn{2}{c}{ Significance } \\
& & Nod $^{+}$ & Nod $^{-}$ & & $\chi^{\mathbf{2}}$ value & $P$ value \\
\hline RISNOD10 & 161 & 123 & 38 & & 0.17 & $P>0.5$ \\
RISNOD19 & 174 & 131 & 43 & & 0.1 & $P>0.9$ \\
RISNOD21 & 184 & 136 & 48 & & 0.12 & $P>0.5$ \\
RISNOD25 & 34 & 28 & 6 & & 0.98 & $P>0.3$ \\
\hline
\end{tabular}

the alleles sym8-1 (R25), sym8-2 (RISNOD10), sym8-3 (RISNOD19), sym8-4 (RISNOD21), and sym8-5 (RISNOD25).

\section{Cloning of SYM8, the pea ortholog of DMII from Medicago truncatula.}

The full-length sequence of the $P$. sativum cDNA homologous to Medicago truncatula DMII was determined for two wildtype pea lines, Sparkle and Finale. This involved a combination of reverse-transcriptase polymerase chain reaction (RTPCR), 5' and $3^{\prime}$ rapid amplification of cDNA ends (RACE), and PCR-based genome walking. Using the derived DNA sequence from the $5^{\prime}$ and $3^{\prime}$ ends, we used RT-PCR to generate cDNA products of 3,188 bp from both Sparkle and Frisson. Their sequences revealed nine nucleotide differences within the coding region, only four of which caused changes in the predicted protein of 895 amino acids. The predicted amino acids tryptophan 61 , leucine 87 , leucine 91 , and glycine 471 in Sparkle are replaced by arginine, isoleucine, valine, and alanine, respectively, in Finale (Fig. 1A). Because the pea sym 8 mutants described above all contain mutations in this cDNA (see below), the pea gene will be referred to as SYM8.

The predicted SYM8 protein from $P$. sativum is $92 \%$ identical to that of $M$. truncatula DMI1 (Fig. 1A) and $87.5 \%$ identical to the L. japonicus POLLUX, but is only $71 \%$ identical to $L$. japonicus CASTOR. SYM8 has the same domain structure as DMI1 and POLLUX (Fig. 1A and B). The amino-termini of the predicted DMI1, SYM8, and POLLUX proteins from the three legumes are the most divergent regions. The program TargetP 1.1 indicates that it is unlikely that the N-terminus of SYM8 contains a chloroplast transit peptide, a mitochondrial targeting peptide, or a secretion peptide (it scored in the lowest reliability class). WoLF PSORT indicated that SYM8 contains three patterns (PLKRTKT, SRKRRP, and RRTNSNKEDVPL KKRVA) that might act as nuclear localization signals between residues 17 and 235 . There are four predicted transmembrane spans in the N-terminal region (residues 135 to 154,205 to 224,266 to 287 , and 31 to -339 ), the first two of which include a putative leucine zipper motif. Database searches using BLAST show that, in addition to the M. truncatula and $L$. japonicus orthologs, SYM8 shows significant sequence similarity to the following predicted proteins or protein fragments: Arabidopsis thaliana Q9LTX4 (69\% identity over 824 amino acids), Oryza sativa Q943J8 (68\% identity over 927 amino acids), Zea mays Q208N2 (89\% identity over 460 amino acids), Glycine max Q1A3R7 (78\% identity over 284 amino acids), and G. max Q1A3R6 (94\% identity over 158 amino acids). The $A$. thaliana and $O$. sativa proteins were predicted to be DMI1 orthologs on the basis of their high protein sequence homology and microsyntenic relationship (Zhu et al. 2006).

A search for conserved domains using rpsblast indicated that the region between amino acids 402 and 590 belongs to the pfam DUF1012 family and also shares approximately $30 \%$ domain similarity with the regulator of conductance of $\mathrm{K}^{+}$(RCK) domain of several bacterial potassium channels, including the structurally characterized MthK channel from Methanobacterium thermoautotrophicum (Anantharaman et al. 2001; Jiang et al. 2002). The RCK domains have been implicated in the regulation of conductance in response to changes in cytoplasmic calcium concentration (Anantharaman et al. 2001; Jiang et al. 2002). The $C$ terminal domain of SYM8 is of unknown function. The similarity between MthK and POLLUX, CASTOR, and DMI1 has been described previously (Ané et al. 2004).

\section{Complementation of the $M$. truncatula dmil mutant by transformation with $P$. sativum SYM8.}

Despite several attempts, we were unable to clone either an intact full-length SYM8 cDNA or the 5' end of the cDNA. 
Therefore, we constructed a hybrid gene containing the $5^{\prime}$ genomic fragment, including two introns, fused to the $3^{\prime} \mathrm{cDNA}$. This hybrid gene, ligated to a 1.6-kb fragment of the DMII promoter (Ané et al. 2004), then was cloned into pCAMBIA1303 to give pCAMBIA-SYM8. Hairy roots of the $d m i 1-1$ mutant C71 transformed with pCAMBIA-SYM8 or pCAMBIADMI1 developed nodules (Table 3), and no significant differences could be detected between the complementation experiments with DMII and SYM8. The presence of bacteria within

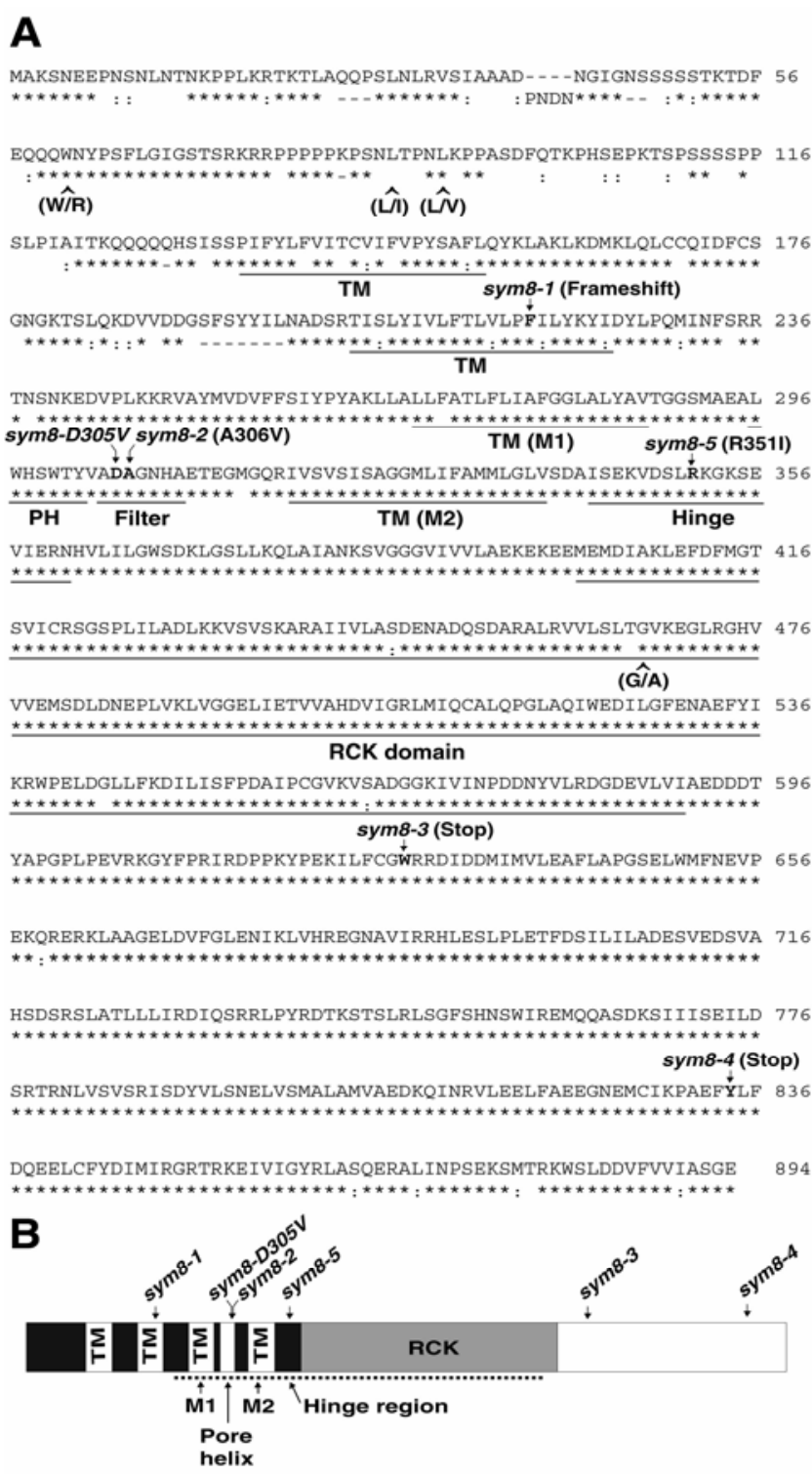

Fig. 1. Sequence and domain structure of Pisum sativum SYM8. A, The sequence shown is derived from Sparkle SYM 8 and amino acid differences in Finale are shown in brackets (W61R, L87I, L91V, and G471A). Residues identical (*) or conserved (:) relative to Medicago truncatula DMI1 are indicated; residues absent from SYM8 or DMI1 are marked (-) and the extra residues (PNDN) in DMI1 are shown at position 42. The positions of the sym 8 mutations are shown in bold; sym8-3 and sym8-4 cause translational stops, sym8- 1 causes a frame shift, sym $8-2$ causes an $\mathrm{A}>\mathrm{V}$ substitution, and sym8-5 causes an $\mathrm{R}>\mathrm{I}$ substitution. The lines indicate the positions of the transmembrane (TM) helices (M1 and M2 corresponding to those in MthK), the filter, the pore helix $(\mathrm{PH})$, the hinge and the regulation of conductance of $\mathrm{K}^{+}(\mathrm{RCK})$ domain. $\mathbf{B}$, The domain structure of SYM8 is shown illustrating the transmembrane helices and the RCK domain (41\% similarity and $27 \%$ identity to the domain regulating $\mathrm{K}^{+}$conductance found in MthK). The positions of the mutations are shown. The dotted line indicates the modeled region of SYM8, which includes transmembrane helices M1 and M2 and the $\mathrm{PH}$ the nodules was demonstrated by staining nodules for rhizobial $\beta$-galactosidase activity and by light microscopy of stained nodule sections (Fig. 2). Nitrogenase activity of the nodules was assayed by acetylene reduction: wild-type nodules produced $3.2 \pm 0.7 \mathrm{nmol}$ ethylene $\mathrm{h}^{-1}$ per nodule while nodules formed by complementation of $d m i 1-1$ by pCAMBIA-SYM8 produced $3.3 \pm 0.6 \mathrm{nmol} \mathrm{h}^{-1}$ per nodule, compared with $3.1 \pm$ $1.7 \mathrm{nmol} \mathrm{h}^{-1}$ per nodule observed with pCAMBIA-DMI1. The ability of wild-type $P$. sativum SYM 8 to complement both nodulation and nitrogen fixation in the dmil-1 mutant confirms that $P$. sativum SYM8 is the ortholog of DMII.

\section{Five independent $P$. sativum sym 8 mutations.}

Full-length $S Y M 8$ cDNA was amplified and sequenced from the mutant pea lines sym8-1 (R25), arising from fast neutron mutagenesis, and sym8-2 (RISNOD10), sym8-3 (RISNOD19), sym8-4 (RISNOD21), and sym8-5 (RISNOD25), which are all EMS mutants. Each line has a different mutation (Fig. 1A). The sym8-1 allele has a 1-bp deletion affecting amino acid F218, and the resulting frame shift produces a predicted product of 229 amino acids. The sym8-3 and sym8-4 alleles carry mutations (nucleotides $\mathrm{G} 2215 \mathrm{~A}$ and T2834A, respectively) changing amino acids W628 and Y834 to stop codons. In sym8-5, the basic residue arginine at 351 is changed to the hydrophobic aliphatic residue, isoleucine; the residue equivalent to R351 is conserved in the homologous gene products

Table 3. Hairy root complementation tests of mutant line Medicago truncatula dmi1-1 for nodulation with DMI1, SYM8, and sym8-D305V

\begin{tabular}{llcc}
\hline $\begin{array}{l}\text { Plant } \\
\text { background }\end{array}$ & Transforming binary plasmid & $\begin{array}{c}\text { GUS } \\
\text { plants }^{\mathbf{a}}\end{array}$ & Nodulated $^{\mathbf{b}}$ \\
\hline dmil-1 & pCAMBIA1303 vector & 19 & 0 \\
dmil-1 & pCAMBIA1303-DMI1 & 39 & 34 \\
dmil-1 & pCAMBIA1303-SYM8 & 44 & 35 \\
dmil-1 & pCAMBIA1303-SYM8-D305V & 30 & 0 \\
\hline
\end{tabular}

a Transformed GUS plants.

${ }^{\mathrm{b}}$ Number of plants nodulated.
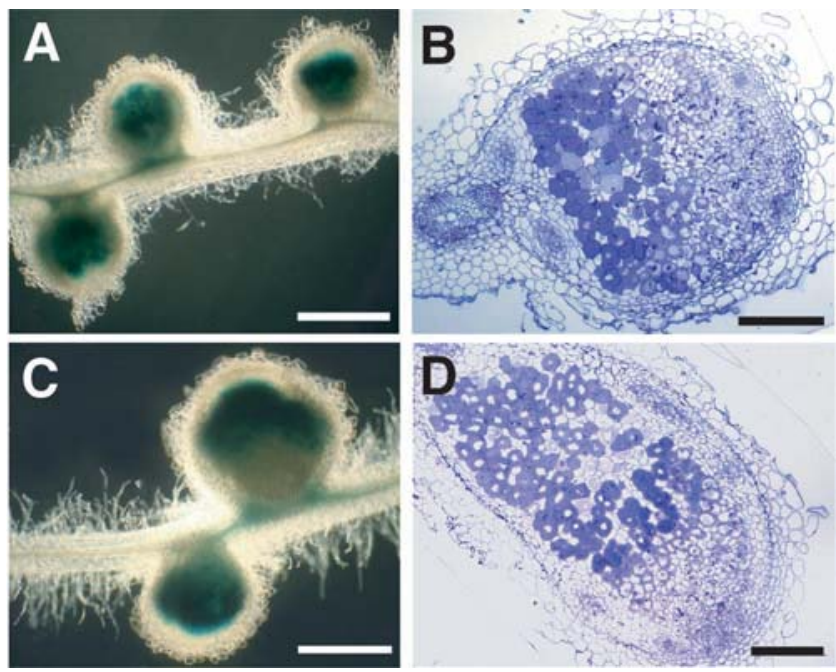

Fig. 2. Complementation of the dmil mutant with SYM8 using Agrobacterium rhizogenes-based transformation. The panel shows representative hairy roots of Medicago truncatula dmil-1 transformed with $\mathbf{A}$ and $\mathbf{B}$, the DMI1 transgene or C and D, SYM 8 transgene, and nodulated with Sinorhizobium meliloti 1021 constitutively expressing lacZ. Nodulation was scored after 15 days. The presence of bacteria within the nodules was determined by $\mathbf{A}$, and $\mathbf{C}$, lac $Z$ staining and $\mathbf{B}$ and $\mathbf{D}$, toluidine blue staining of sectioned nodules. No nodules were seen when the dmil-1 mutant was transformed with the binary vector pCAMBIA-1303 alone. Scale bars: A and $\mathrm{C}, 1 \mathrm{~mm}$ and $\mathrm{B}$ and $\mathrm{D}, 0.2 \mathrm{~mm}$. 
predicted in M. truncatula, L. japonicus, $O$. sativa, and $A$. thaliana. In sym8-2, the alanine at 306 is replaced by valine; the residue equivalent to A306 is conserved in M. truncatula DMII but is present as serine in CASTOR and POLLUX in $L$. japonicus, and in the homologous gene products of $O$. sativa and $A$. thaliana. This suggests that this position can tolerate a small residue such as alanine or serine but the relatively large residue, valine, can interfere with function.

The lack of function of sym8 in the sym8-2 and sym8-5 mutants is likely to be due to the substitution of critical residues, but also could be caused if these missense mutations result in the proteins being destabilized and degraded by proteases. To determine whether stable mutant proteins were produced following hairy root transformation, the pCAMBIA-SYM8 construct was modified such that the residues ELDYKDDDDK would be added to the C-terminus of the SYM8 protein. This includes an antigenic FLAG-tag and the construct was called pCAMBIA-SYM8-FLAG. The sym8-2 and sym $8-5$ mutations then were introduced into that construct to produce pCAMBIA-sym8-2-FLAG and pCAMBIA-sym8-5-FLAG. The dmil1 mutant was transformed with each of these constructs and with the vector control pCAMBIA-1303 using Agrobacterium rhizogenes-mediated hairy root transformation. Protein was extracted from transformed roots, separated by sodium dodecyl sulfate polyacrylamide gel electrophoresis, and transferred to a polyvinylidene difluoride (PVDF) membrane. Antiserum to the FLAG-tag recognized several bands, including a protein of approximately $100 \mathrm{kDa}$, the expected size of the tagged DMI1 protein. Similar bands of $100 \mathrm{kDa}$ were seen with the hairy roots transformed with the pCAMBIA-sym8-2-FLAG and pCAMBIA-sym8-5-FLAG constructs, but not with the vector control pCAMBIA-1303 (Fig. 3). These results are consistent with the sym8-2 and sym $8-5$ mutants producing relatively stable but nonfunctional gene products.

\section{Structure-function implications}

of the sym $8-2$ and sym $8-5$ mutations.

In order to try to understand why the sym8-2 (A306V) and sym8-5 (R351I) mutations block the function of the SYM8 protein, we developed a homology model of SYM8, using as

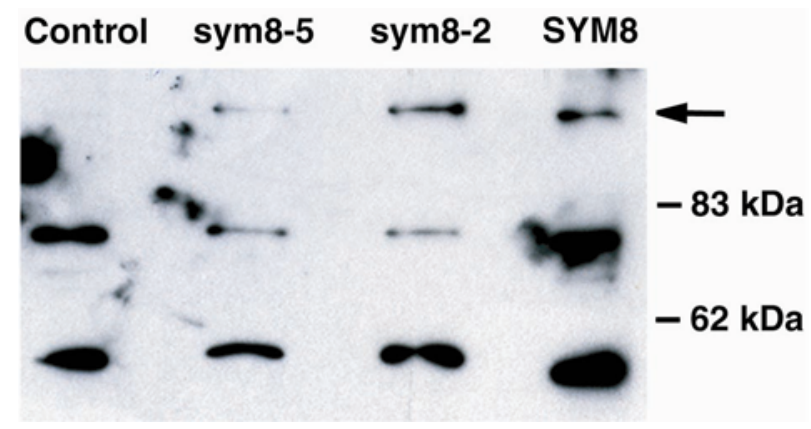

Fig. 3. Western blot of FLAG-tagged SYM8, sym8-2, and sym8-5 proteins expressed in roots. Proteins were extracted from Agrobacterium rhizogenesinduced hairy roots of the Medicago truncatula dmil-1 mutant transformed with pCAMBIA-1303 (Control), pCAMBIA-SYM8-5-FLAG (sym8-5), pCAMBIA-SYM8-2-FLAG (sym8-2), and wild-type pCAMBIA-SYM8FLAG (SYM8). For each, approximately $20 \mu \mathrm{g}$ of root proteins were separated by sodium dodecyl sulfate polyacrylamide gel electrophoresis and then transferred to a polyvinylidene difluoride membrane. The membrane was incubated with a rabbit anti-FLAG polyclonal antibody and immunolabeled proteins were detected using enhanced chemiluminescence. The migration positions of marker proteins identified on the gel are indicated and the arrow points to the $100-\mathrm{kDa}$ protein predicted to be the FLAGtagged derivatives of SYM8, sym8-2, and sym8-5. The other proteins reacting with the antiserum are independent of the FLAG-tagged SYM8 alleles and can be considered as loading controls.
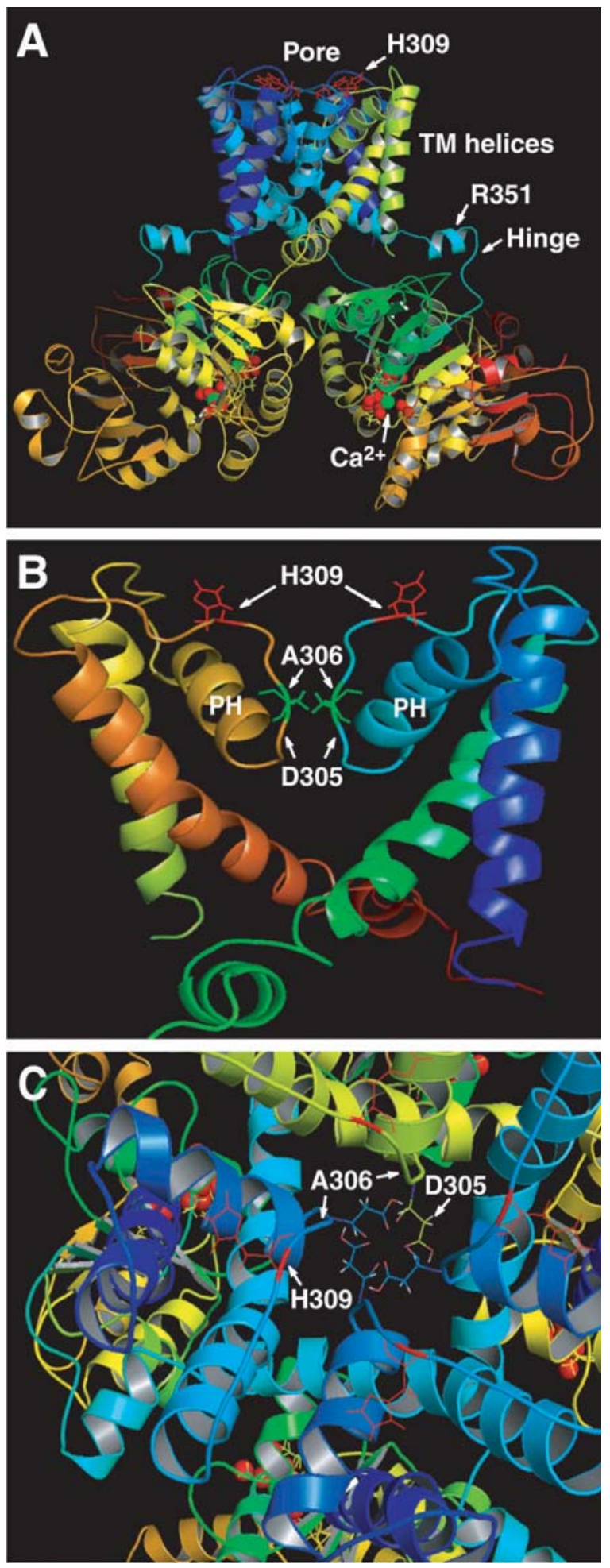

Fig. 4. Model of a SYM8 channel. A, The predicted ribbon structural model shows a tetramer with the transmembrane domain showing transmembrane (TM) helices with histidine (H309) residues (in red) at the top of the predicted pore. The predicted gating-ring region is shown with four bound $\mathrm{Ca}^{2+}$ atoms shown in green and their potential oxygen ligands shown in red. A hinge region linking the pore with the gating ring has been modeled and the position of arginine 351 mutated in sym8-5 is indicated. $\mathbf{B}$, The pore region oriented as above is shown in more detail and, for clarity, two of the subunits have been omitted so that two of the pore helices (PH) equivalent to those in MthK can be seen more clearly. The side groups of alanine 306 (A306) that is mutated in sym8-2 are included and the side groups of $\mathrm{H} 309$ are included for reference. $\mathbf{C}$, The model as in A viewed from the top showing the pore region. The locations of A306 and H309 are indicated and the side groups of D305 are shown extending into the pore. 
a template the crystal structure of the $\mathrm{Ca}^{2+}$-gated potassium channel MthK from Methanobacterium thermoautotrophicum (Jiang et al. 2002). A protein structure similarity search using FUGUE revealed that the region of SYM8 between amino acids 262 and 604 shows significant structural matches to MthK. The MthK channel exhibits two biophysical properties: selective ion conductance and the ability to gate-open in response to an appropriate stimulus (e.g., using the free energy of intracellular $\mathrm{Ca}^{2+}$ binding to perform mechanical work to open the pore) (Jiang et al. 2002). The SYM8 model was generated using InsightII, the molecular modeling and graphics package developed by Accelrys in conjunction with sequence alignments determined using the on-line tools 'phyre' and 'Muscle' (Edgar 2004). The full details of the modeling and resulting predicted structural characteristics will be published elsewhere; however, the key aspects of the model will be presented here in relation to the predicted structural context of the amino acid substitutions in the sym8-2 and sym8-5 mutants.

Each of the four subunits of MthK contributing to the pore region contained two transmembrane helices (M1 and M2) and a pore-forming helix $(\mathrm{PH})$ that extends into the membrane where the filter region is located (Jiang et al. 2002). These 12 helices are conserved in our ribbon model of a tetrameric SYM8 (Fig. 4A) and, for clarity, we have shown a detailed model of two subunits of the helical regions that contribute to the pore (Fig. 4B). The helices are linked via a predicted hinge region (Fig. 4A) to a membrane-associated domain that is predicted to form part of the channel-gating ring. In this model, we have not incorporated the $\mathrm{N}$-terminal domain (residues 1 to 261), including two predicted transmembrane domains that are $\mathrm{N}$-terminal to the transmembrane helix equivalent to M1 (Fig. 1B). Also excluded are residues 605 to 894, which we were unable to model. These regions were omitted because neither is present in MthK. Although we have shown SYM8 as a tetramer of the pore and gating ring regions (Fig. 4A), in the MthK channel the gating-ring region is octameric, containing another four subunits made from an alternative translation start of $M t h K$, such that these subunits contribute to the gating ring but not to the pore.

The partial model of the predicted SYM8 channel (Fig. 4A) highlights several key points with regard to the predicted structure. There are eight transmembrane-spanning helices surrounding a predicted central pore region formed by four poreforming helices, which extend into (but not across) the membrane. The filter region in the MthK central pore region has a classic conserved sequence TVGYG (Heginbotham et al. 1994) that confers specificity to $\mathrm{K}^{+}$. In SYM8, this is not present and, in the equivalent filter region of the SYM8 model, the residues are ADAGN (Figs. 1A and 4B). The fact that the loss-of-function sym8-2 allele replaces the central alanine (A306) with valine gives supporting evidence that the region we model as part of the pore is critical for function. Figure 4B shows the location of A306 in relation to the helices and Figure 4C shows a top view looking down through the pore region, illustrating the location of the A306V mutation.

It is clear from Figure 4B that the aspartate (D305) residues appear to form a charged ring within the membrane (Fig. 4B and $\mathrm{C}$ ). The predicted presence of charged residues in such a hydrophobic membrane location is highly significant. To test the importance of this aspartate, we altered it to valine, which is uncharged but structurally similar, and is the same residue that is present at the predicted equivalent structural location in the MthK pore region. This mutated gene was cloned into the transformation vector to make pCAMBIASYM8-D305V and tested for function by complementation of the Medicago truncatula dmil-1 mutation using trans- formed hairy roots. No complementation was observed (Table 3), showing that this mutation leads to loss of function. Therefore, we conclude that the residues ADAGN in SYM8 are likely to play a key role in SYM8 function with the aspartate conferring cation selectivity.

At the top of the predicted channel is a relatively open ring of histidine residues (Fig. 4, H309; shown in red) adjacent to the predicted central pore region and the size of this ring fits well with the calculated size of a hydrated cation such as $\mathrm{K}^{+}$. Therefore, this histidine ring might play a role in the hydration or dehydration of cations moving out of or into the channel. As described by Consiglio and associates (2003), the presence of such a residue can strongly influence the $\mathrm{K}^{+}$selectivity and conductance of a $\mathrm{K}^{+}$channel by affecting the exit of hydrated ions from the channel. This would imply that cations (most likely $\mathrm{K}^{+}$) primarily move up through the channel as drawn in Figure 4A. The net function of such a channel could be to catalyze membrane depolarization or hyperpolarization which, in turn, could induce the opening of a calcium channel.

Our model shows that the predicted membrane pore region of SYM8 is linked to a predicted $\mathrm{Ca}^{2+}$ binding domain that is membrane associated (Fig. 4C). We have shown a predicted location of $\mathrm{Ca}^{2+}$ which is close to that observed in the MthK crystal structure; however, this will require further analysis to determine whether there is $\mathrm{Ca}^{2+}$ binding and, if so, which residues are involved in $\mathrm{Ca}^{2+}$ binding. In $\mathrm{MthK}, \mathrm{Ca}^{2+}$ binding is thought to cause a protein conformational change that induces opening of the pore (Jiang et al. 2002). Linking the pore domain and gating ring in MthK is a disordered region (extending from residues L98 to R116 in MthK) which could not be resolved in the crystal structure. The equivalent region in SYM8 corresponds to residues I343-N361. Significantly, the sym8-5 allele causes arginine 351 in this domain (Fig. 4A) to be replaced with isoleucine. Therefore, it seems possible that this R351I loss-of-function mutation in the proposed hinge region could prevent conformational changes in the gating region from being transmitted to the pore region. We have modeled this hinge region based on related sequences and structural optimization.

\section{DISCUSSION}

The data presented here confirm the earlier prediction (Ané et al. 2004), based on synteny and phenotypic similarities, that SYM8 in pea is the ortholog of M. truncatula DMII. Although the homology between pea and $M$. truncatula is apparent, the comparison with $L$. japonicus is less clear because this legume has two very similar genes, CASTOR and POLLUX (ImaizumiAnraku et al. 2005). Both these proteins show similarity to SYM8 and DMI1. SYM8 is most likely to be orthologous to POLLUX based on similarity and map location. We could complement the dmil-1 mutant of M. truncatula with cloned SYM8, confirming that SYM8 and DMII are orthologous. DMI1 was found at the nuclear envelope (Riely et al. 2007), whereas POLLUX (like its partner, CASTOR) has been reported to be associated with plastids (Imaizumi-Anraku et al. 2005). The N-terminal sequence of SYM8 is unlikely to be a plastid targeting sequence based on the prediction program TargetP 1.1 (score of 5), and a similar prediction was obtained using the DMI1 sequence. Like DMI1 (Riely et al. 2007), SYM8 has three potential nuclear localization signals at the N-terminus, and these also are conserved in POLLUX from $L$. japonicus (GenBank accession AB162158). However, more detailed biochemical work will be required to test the proposed nuclear location of SYM8.

It is not clear whether the SYM8 and DMI1 proteins function only in symbiosis or play another physiological role. 
Apart from the defects in rhizobial and mycorrhizal symbioses, we have observed no other phenotypes in sym 8 mutants. Based on similarities, it seems likely that orthologous genes are found in nonlegumes (Zhu et al. 2006) and, in principle, these could function during mycorrhization. However, if the similar gene in Arabidopsis thaliana is a true ortholog (Zhu et al. 2006), then this implies that SYM8 and DMI1 may have another role, because there is no mycorrhizal symbiosis in Arabidopsis.

A key question is how DMI1 and SYM8 function. Mutations in their genes block Nod-factor-induced $\mathrm{Ca}^{2+}$ spiking (Wais et al. 2000; Walker et al. 2000) and the subsequent induction of early nodulation genes (Mitra et al. 2004). The possibility that DMI1 is a channel required for calcium movement during the oscillations must be considered. However, this seems unlikely based on the observation that Mastoparan can induce calcium spiking in dmil mutants (Peiter et al. in press), suggesting that DMI1 is not essential for the oscillations in calcium. An alternative model is that SYM8 and DMI1 form a channel that is required for the activation of calcium spiking.

It was recognized previously that DMI1, CASTOR, and POLLUX are likely to form ion channels (Ané et al. 2004; Imaizumi-Anraku et al. 2005); however, the amino acids at the filter region showed no sequence conservation with the filter region of the characterized $\mathrm{Ca}^{2+}$-gated $\mathrm{K}^{+}$channel (MthK) from Methanobacterium thermoautotrophicum (Jiang et al. 2002). The structural model predicted here indicates the presence of a negatively charged aspartate residue at the choke of the predicted filter region (VADAGNH) within the membrane, strongly suggesting cation selectivity of the channel. We also identified a ring of histidine residues on the outer edge of the pore-forming helices and, based on work with animal $\mathrm{K}^{+}$-selective channels (Consiglio et al. 2003), we propose that these histidine residues may contribute to cation selectivity.

Replacing the aspartate with valine abolished function, adding substance to our prediction that the aspartate is important for ion movement. One of the two other missense mutations (alanine 306 to valine) we identified is highly likely to disrupt the structural integrity of the filter region because it is adjacent to the aspartate in SYM8. Significantly, the castor- 2 allele in L. japonicus is a loss-of-function caused by mutation of alanine to threonine on the other side of this aspartate residue (Imaizumi-Anraku et al. 2005. This alanine residue is located at the bottom of the filter region adjacent to the $\mathrm{PH}$ in the model. In the sym8-D305V, sym $8-2$, and castor- 2 mutants, we assume that function is lost because the channel activity is blocked by the mutations, although proving this will require biochemical work with isolated proteins to identify which ions move through the channel and the effects of these mutations on that movement.

The other missense mutation we identified in the sym8-5 mutant was arginine 351 to isoleucine, in what we predicted to be a hinge region joining the transmembrane helices to the large calcium-binding domain that is predicted to be adjacent to (but not spanning) the membrane. We propose that this mutation may prevent activation of the pore following a conformational change in the predicted gating-ring region. In our model, based on the MthK crystal structure, we identify potential $\mathrm{Ca}^{2+}$-binding pockets in this region; therefore, we speculate that binding of $\mathrm{Ca}^{2+}$ might gate the channel in a manner analogous to that seen with MthK (Jiang et al. 2002). The structure of the MthK channel is somewhat unusual because it is octameric, made up of two different translation products from the same gene. Thus, four subunits of a full-length MthK form a transmembrane channel with membrane-associated $\mathrm{Ca}^{2+}$-binding domains and four additional $\mathrm{Ca}^{2+}$-binding domains in the complex are derived from a partial gene product (Jiang et al. 2002).
Clearly, it will require biochemical work to test the ion selectivity of the predicted SYM8 and DMI1 channels and the related CASTOR/POLLUX channel. However, the existing amino acid substitutions, together with our model, allow some structure-function predictions to be made and to direct mutagenesis, targeting residues that probably contribute to the filter region, the $\mathrm{Ca}^{2+}$-binding pocket, and the hinge region that we propose may act to transfer a conformational change from the gating ring to the pore. One possibility is that SYM8 and DMI1 form cation channels and that activation of the channel by binding of a signaling ligand could cause it to open, creating a change in polarization of the nuclear membrane. Such a polarization-induced change could activate the opening of a separate $\mathrm{Ca}^{2+}$ channel, because both depolarization-activated and hyperpolarization-activated channels are found in plants (White 2000). The resulting changing $\mathrm{Ca}^{2+}$ levels then could have a feedback effect on the activity of the SYM8/DMI1 channel. The observation that the $\mathrm{Ca}^{2+}$ spikes occur around the nuclear region (Erhardt et al. 1996; Walker et al. 2000) fits with a model of SYM8/DMI1 activation of a channel in the nuclear envelope. Therefore, we conclude that the requirement of SYM8, DMI1, and CASTOR/POLLUX for Nod-factorinduced $\mathrm{Ca}^{2+}$ spiking could be to induce a change in membrane polarization that activates the opening of an unidentified $\mathrm{Ca}^{2+}$ channel, which is required for $\mathrm{Ca}^{2+}$ spiking.

\section{MATERIALS AND METHODS}

Growth, genetic crosses, and phenotypic scoring of pea.

$P$. sativum cultivars used were Frisson, Finale, and Sparkle. For scoring of nodulation, mutant lines and their progeny were grown in the greenhouse under nitrogen limitation. Mutant R25 is derived from Sparkle. Mutants RISNOD10, RISNOD19, RISNOD21, and RISNOD25 are derived from Finale. The screening procedure for establishing root nodulation was as described by Duc and Messager (1989) and Duc and associates (1989).

\section{Cloning and sequence analysis of SYM8.}

For isolation of RNA, pea seed were surface sterilized and germinated for 8 days at room temperature on water agar plates. Approximately 10 root tips of 1 to $2 \mathrm{~cm}(100 \mathrm{mg})$ were excised from each wild type or mutant and RNA was extracted using an RNeasy plant mini kit (Qiagen Ltd., Crawley, U.K.). cDNA was synthesized using $1 \mu \mathrm{g}$ of total RNA and Superscript II reverse transcriptase (Invitrogen Ltd., Renfrew, U.K.) following the manufacturer's recommended conditions. Genomic DNA was isolated from pea leaves using a Zenogene plant DNA purification kit (Zenon Bio Kft., Szeged, Hungary)

Initially, a 1,490-bp cDNA fragment of pea SYM8 was amplified with primers to $M$. truncatula DMI1 (GTGCTTGCAG AAAAGGAAAAGGAG and CTATTCCTTTCTTCTGTC AT TCACCTGAGG) using oligo-dT primed pea cDNA as template and an annealing temperature of $45^{\circ} \mathrm{C}$. The resulting PCR product was sequenced. Nested primers (CTTCTTTAAG TCAGCCAGTATAAGTGGAC and CATGAAATCAAATTCT AGCTTGG) designed from within the amplified region were used for 5' RACE using the Invitrogen GeneRacer Kit to generate a product of $734 \mathrm{bp}$. The primer CTTCAGATAAATC AATTATAATTAGTG was used for $3^{\prime}$ RACE and generated a $3^{\prime}$ product of $793 \mathrm{bp}$. Primers derived from the 734- and 793bp RACE products ( $5^{\prime}$ primer CTGAATGCAGATAGTAGAA CAATTTC and $3^{\prime}$ primer CGAATTCACTAGTGATTGAA AC AAAAG) were used to amplify a 2,584-bp cDNA product, which was sequenced.

The $5^{\prime}$ end of SYM8 was amplified from a pea (cv. Sparkle) GenomeWalker library (BD Bioscience, Oxford) using the 
nested primers CTGCGTCGGCTACATAAGTCCATGAATG, CAACGCTAAACCACCAAATGCTATAAG, TTATAAGCTC GTAAGAAAGTGACAATAACC, and CAGTCTCGGCCGAT GGCAGATAAGCTAG with an EcoRV library, and the primer GAGGTGGCGGTCTTCTTTTTCTG with a DraI library. Finally, a 1,945-bp fragment was amplified and it contained two introns (100 and $640 \mathrm{bp}$ ). Sequencing of this fragment located the predicted translational start site, which matched perfectly with that of $M$. truncatula DMII. A full-length SYM 8 cDNA fragment of $3,188 \mathrm{bp}$ was amplified using primer pair 5' primer GAGGTACCTCGCTCACTCGTTCACTAACTAATC, which lies 39 bp upstream of the predicted translational start and which also incorporates a KpnI site (shown in bold), and 3' primer TTGAATTCGAAACAAAAGTGAATTTTATATAT AGACC, which lies 430 bp downstream of the predicted translational stop and introduces an EcoRI site (shown in bold) at the $3^{\prime}$ end. The same primers were used to amplify full-length cDNA from all five sym 8 mutants. The PCR products were purified using a QIAQUICK PCR purification kit (Qiagen Ltd.) and sequenced directly using a Big Dye terminator version 3.1 kit (Applied Biosystems, Reading, U.K.). Sequences were analyzed using GCG programs and BLAST. CLUSTAL $\mathrm{W}$ was used for multiple alignment. The target peptide and transmembrane regions were predicted by TargetP version 1.1, WoLF PSORT, and TMpred. Domain and structure analysis was performed by FUGUE v2.0.

When we tried to clone the full-length cDNA fragment as a $K p n I-E c o$ RI fragment or the $5^{\prime}$ region as a KpnI-XhoI fragment, the resulting clones always contained deletions. Therefore, to generate a stable full-length clone of $S Y M 8$, we separately generated a $5^{\prime}$ genomic fragment and a $3^{\prime}$ cDNA fragment. The 3' PCR product of 2,584 bp was digested to 2,450 bp with XhoI and EcoRI and then cloned between the XhoI and EcoRI sites of pBluescript-SKII (Stratagene, Cambridge) to form pIJ9868. The $5^{\prime}$ region, including introns and with an introduced $K p n I$ site, was amplified from genomic DNA as a 1,945-bp fragment, (primer pair GAGGTACCTCGCTCACTC GTTCACTAACTAATC and CAAATGCTATAAGAAAGAGA GTTGCAAAAAGAAG), which contains two introns of 100 and $640 \mathrm{bp}$. This was digested with $K p n \mathrm{I}$ and XhoI to 1,489 bp and ligated into pIJ9868 cut with KpnI and XhoI to give plasmid pIJ9870, in which the $5^{\prime}$ end of SYM8 containing two introns is joined at a native $\mathrm{XhoI}$ site with the $3^{\prime} \mathrm{cDNA}$ fragment. The resulting KpnI-EcoRI fragment in pIJ9870 is equivalent to 3,188 bp of cDNA but actually includes an additional 740 bp of intron DNA.

\section{Construction of binary vectors expressing SYMS and modified derivatives.}

The $M$. truncatula DMII promoter was amplified from pCAMBIA-DMI1 (Ané et al. 2004) as a 1.6-kb fragment containing introduced EcoRI and KpnI sites as indicated (boldface) on the 5' and 3' primers: GGGGTACCGAATTCCACA GCGAAGAAAAGG and GGGGTACCTTTCTTTTCTT CAC ACTTTTAAA. The product was cloned into KpnI-linearized pIJ9870 containing full-length SYM8 cDNA to give plasmid pIJ9880. A 5.8-kb EcoRI fragment containing full-length SYM8 under the control of the DMII promoter was excised from pIJ9880 and cloned into binary vector pCAMBIA-1303 (AF234299) to give pCAMBIA-SYM8. To generate pCAMBIA-SYM8-D305V, the primer pair 5' ATGGACTTATGTAG CCGTCGCAGGAAATCATGCTG $3^{\prime}$ and 5' CAGCATGATT TCCTGCGACGGCTACATAAGTCCAT 3' was used for sitedirected mutagenesis (QuikChange II XL; Stratagene) of pCAMBIA-SYM8 to change aspartate 305 to valine. pCAMBIA-1303 also carries uidA under the constitutive 35S promoter; therefore, it is possible to determine which roots have been transformed by staining with 5-bromo-4-chloro-3-indoyl- $\beta$-Dglucoronide as described by Jefferson (1987).

To generate a C-terminal FLAG-tagged SYM8 gene, the 3' end of the coding region of Sym8 in pIJ9868 was mutagenized to introduce a SacI site (boldface) and to remove the translational stop codon using a QuikChange II XL site-directed mutagenesis kit (Stratagene) and primer pair 5' GTTATTGC CTCAGGAGAAGAGCTCGAAAGAATAGTATAGTTATTAT TC $3^{\prime}$ and 5' GAATAATACTATACTATTCTTTCAGAGCTCT TCTCCTGAGGCAATAAC 3' to give pIJ11008. This then was used as a template for amplification with a primer encoding a FLAG Tag (shown in italics) followed by a translational stop GAAGAGCTCGATTATAAAGATGAT GACGATAAGTGAAA GAATAGTATAGTATTATTCTTG and the M13 reverse primer. The resulting 500-bp fragment was digested with SacI and cloned into pIJ11008 to give pIJ11009. An 800-bp XbaI fragment containing the FLAG tag from pIJ110099 was excised and used to replace the equivalent $X b a$ I fragment in pCAMBIASYM8 to give pCAMBIA-SYM8-FLAG. In this construct, SYM8 has the extra peptide ELDYKDDDDK at the $\mathrm{C}$ terminus to give a protein of approximately $100 \mathrm{kDa}$. pCAMBIASYM8-FLAG was used as template for site-directed mutagenesis (QuikChange) to recreate the sym8-2 and sym8-5 mutations, producing the two mutant forms pCAMBIA-sym82-FLAG and pCAMBIA-sym8-5-FLAG using the primer pairs 5' GACTTATGTAGCCGACGTAGGAAATCATGCTG 3' and 5' CAGCATGATTTCCTAC GTCGGCTACATAAGTC $3^{\prime}$ and 5' GAGAAGGTTGATTCACTTATAAAAGGGAGAGTG AA GTG 3' and 5' CACTTCACTCTTCCCTTTTATAAGTGAAT CAACCTTCTC 3', respectively; the mutational changes are shown in boldface. All derivatives of SYM8 used for complementation experiments were confirmed by DNA sequencing.

\section{Complementation tests for nodulation.}

pCAMBIA-1303, pCAMBIA-DMI1, pCAMBIA-SYM8, and pCAMBIA-SYM8-D305V were transferred into Agrobacterium rhizogenes QUA1 (Quandt et al. 1993) and used to transform M. truncatula dmi1-1 mutant C71 (Ané et al. 2004) using hairy root transformation (Boisson-Dernier et al. 2001). Following A. rhizogenes inoculation, seedlings were grown on modified Fahraeus (Boisson-Dernier et al. 2001) medium for 3 weeks, then transferred to CYG growth pouches (16.5 by 17.5 $\mathrm{cm}$; Mega International, Minneapolis, MN, U.S.A.) inoculated with Sinorhizobium meliloti 1021/pXLD4 that expresses lacZ from the hemA promoter. After 15 days, nodulation was scored and $\beta$-glucuronidase staining was used to identify transformed roots. Nodules were stained with X-gal to detect the presence of bacteria as described by Walker and Downie (2000). For sectioning, unstained nodules were cut in half longitudinally and fixed, sectioned, and stained as described previously (Lodwig et al. 2005). Photographs were taken on a Zeiss axiophot microscope with a Canon EOS 10D digital camera. Acetylene reduction was assayed using nodules excised 21 days after inoculation as described by Somasegaran and Hoben (1994). Results were expressed as nanomoles ethylene produced per hour per nodule.

\section{Protein extraction, gel electrophoresis, and immunodetection.}

Proteins were extracted from transformed hairy roots (Ramos and Bisseling 2003) and samples of approximately 20 $\mu \mathrm{g}$ were separated by electrophoresis through a $10 \%$ polyacrylamide gel, then transferred by electroblotting to a PVDF membrane. The membrane was incubated with a rabbit antiFLAG polyclonal antibody (Sigma-Aldrich, Poole, U.K.) and immunolabeled proteins were detected using ECL (Amersham Biosciences, Buckingshamshire, U.K.). 


\section{ACKNOWLEDGMENTS}

This study was supported by a Biotechnology and Biological Sciences Research Council grant-in-aid and a European Union Marie Curie PhD Fellowship (RTN-CT-2003-505227) (to A. B. Heckmann) via the INTEGRAL network. We thank S. Bunnewell for her expert work on embedding and sectioning nodules for microscopy; D. Cook, University of CaliforniaDavis, for the gift of pCAMBIA-DMI1; M. Ambrose for making available pea seed; K. C. Engvild for providing M3 progenies of pea nodulation mutants; and T. Larue, M. Sagan, F. Moussy, and H. De Larambergue for their contributions to the complementation analyses. We thank G. Oldroyd and members of our lab for stimulating discussions and M. Charpentier for advice.

\section{LITERATURE CITED}

Anantharaman, V., Koonin, E. V., and Aravind, L. 2001. Regulatory potential, phyletic distribution and evolution of ancient, intracellular smallmolecule-binding domains. J. Mol. Biol. 307:1271-1292.

Ané, J. M., Levy, J., Thoquet, P., Kulikova, O., de Billy, F., Penmetsa, V., Kim, D. J., Debelle, F., Rosenberg, C., Cook, D. R., Bisseling, T., Huguet, T., and Denarie, J. 2002. Genetic and cytogenetic mapping of DMI1, DMI2, and DMI3 genes of Medicago truncatula involved in nod factor transduction, nodulation, and mycorrhization. Mol. PlantMicrobe Interact. 15:1108-1118.

Ané, J. M., Kiss, G. B., Riely, B. K., Penmetsa, R. V., Oldroyd, G. E. D., Ayax, C., Levy, J., Debelle, F., Baek, J. M., Kalo, P., Rosenberg, C., Roe, B. A., Long, S. R., Denarie, J., and Cook, D. R. 2004. Medicago truncatula DMI1 required for bacterial and fungal symbioses in legumes. Science 303:1364-1367.

Arrighi, J. F., Barre, A., Ben Amor, B., Bersoult, A., Soriano, L. C., Mirabella, R., de Carvalho-Niebel, F., Journet, E. P., Gherardi, M., Huguet, T., Geurts, R., Denarie, J, Rouge, P., and Gough, C. 2006. The Medicago truncatula LysM motif-receptor-like kinase gene family includes NFP and new nodule-expressed genes. Plant Physiol. 142:265279.

Balaji, B., Ba, A. M., Larue, T. A., Tepfer, D., and Piche, Y. 1994. Pisum sativum mutants insensitive to nodulation are also insensitive to invasion in-vitro by the mycorrhizal fungus, Gigaspora margarita. Plant Sci. 102:195-203.

Boisson-Dernier, A., Chabaud, M., Garcia, F., Becard, G., Rosenberg, C., and Barker, D. G. 2001. Agrobacterium rhizogenes-transformed roots of Medicago truncatula for the study of nitrogen-fixing and endomycorrhizal symbiotic associations. Mol. Plant-Microbe Interact. 14:695700 .

Cardenas, L., Vidali, L., Dominguez, J., Perez, H., Sanchez, F., Hepler, P. K., and Quinto, C. 1998. Rearrangement of actin microfilaments in plant root hairs responding to Rhizobium etli nodulation signals. Plant Physiol. 116:871-877.

Consiglio, J. F., Andalib, P., and Korn, S. J. 2003. Influence of pore residues on permeation properties in the Kv2.1 potassium channel. Evidence for a selective functional interaction of $\mathrm{K}^{+}$with the outer vestibule. J. Gen. Physiol. 121:111-124.

Duc, G., and Messager, A. 1989. Mutagenesis of pea (Pisum sativum L.) and the isolation of mutants for nodulation and nitrogen-fixation. Plant Sci. 60:207-213.

Duc, G., Trouvelot, A., Ginaninazzi-Pearson, V., and Gianinazzi, S. 1989. First report of non-mycorrhizal plant mutants $\left(\mathrm{Myc}^{-}\right)$obtained in pea (Pisum sativum L.) and faba bean (Vicia faba L.). Plant Sci. 60:215222.

Edgar, R. C. 2004. MUSCLE: multiple sequence alignment with high accuracy and high throughput. Nucleic Acids Res. 32:1792-1797.

Ehrhardt, D. W., Wais, R., and Long, S. R. 1996. Calcium spiking in plant root hairs responding to Rhizobium nodulation signals. Cell 85:673681.

Endre, G., Kereszt, A., Kevei, Z., Mihacea, S., Kalo, P., and Kiss, G. B. 2002. A receptor kinase gene regulating symbiotic nodule development. Nature 417:962-966.

Engvild, K. J. 1987. Nodulation and nitrogen fixation mutants of pea (Pisum sativum). Theor. Appl. Genet. 74:711-713.

Geurts, R., Heidstra, R., Hadri, A. E., Downie, J. A., Franssen, H., vanKammen, A., and Bisseling, T. 1997. Sym2 of pea is involved in a nodulation factor-perception mechanism that controls the infection process in the epidermis. Plant Physiol. 115:351-359.

Guinel, F. C., and Geil, R. D. 2002. A model for the development of the rhizobial and arbuscular mycorrhizal symbioses in legumes and its use to understand the roles of ethylene in the establishment of these two symbioses. Can. J. Bot. 80:695-720.

Harris, J. M., Wais, R., and Long, S. R. 2003. Rhizobium-induced calcium spiking in Lotus japonicus. Mol. Plant-Microbe Interact. 16:335-341.

Harrison, M. J. 1999. Molecular and cellular aspects of the arbuscular mycorrhizal symbiosis. Annu. Rev. Plant Physiol. Plant Mol. Biol. 50:361389.

Heginbotham, L., Lu, Z., Abramson, T., and MacKinnon, R. 1994. Mutations in the $\mathrm{K}^{+}$channel signature sequence. Biophys. J. 66:1061-1067.

Imaizumi-Anraku, H., Takeda, N., Charpentier, M., Perry, J., Miwa, H., Umehara, Y., Kouchi, H., Murakami, Y., Mulder, L., Vickers, K., Pike, J., Downie, J. A., Wang, T., Sato, S., Asamizu, E., Tabata, S., Yoshikawa, M., Murooka, Y., Wu, G. J., Kawaguchi, M., Kawasaki, S., Parniske, M., and Hayashi, M. 2005. Plastid proteins crucial for symbiotic fungal and bacterial entry into plant roots. Nature 433:527-531.

Jefferson, R. A. 1987. Assaying chimeric genes in plants: the GUS gene fusion system. Plant Mol. Biol. Rep. 5:387-405.

Jiang, Y. X., Lee, A., Chen, J. Y., Cadene, M., Chait, B. T., and MacKinnon, R. 2002. Crystal structure and mechanism of a calciumgated potassium channel. Nature 417:515-522.

Kanamori, N., Madsen, L. H., Radutoiu, S., Frantescu, M., Quistgaard, E. M. H., Miwa, H., Downie, J. A., James, E. K., Felle, H. H., Haaning, L. L., Jensen, T. H., Sato, S., Nakamura, Y., Tabata, S., Sandal, N., and Stougaard, J. 2006. A nucleoporin is required for induction of $\mathrm{Ca}^{2+}$ spiking in legume nodule development and essential for rhizobial and fungal symbiosis. Proc. Natl. Acad. Sci. U.S.A. 103:359-364.

Kistner, C., and Parniske, M. 2002. Evolution of signal transduction in intracellular symbiosis. Trends Plant Sci. 7:511-518.

Kneen, B. E., Weeden, N. F., and Larue, T. A. 1994. Non-nodulating mutants of Pisum sativum (L.) cv. Sparkle. J. Hered. 85:129-133.

Levy, J., Bres, C., Geurts, R., Chalhoub, B., Kulikova, O., Duc, G., Journet, E. P., Ane, J. M., Lauber, E., Bisseling, T., Denarie, J., Rosenberg, C., and Debelle, F. 2004. A putative $\mathrm{Ca}^{2+}$ and calmodulin-dependent protein kinase required for bacterial and fungal symbioses. Science 303:1361-1364.

Limpens, E., Franken, C., Smit, P., Willemse, J., Bisseling, T., and Geurts, R. 2003. LysM domain receptor kinases regulating rhizobial Nod factor-induced infection. Science 302:630-633.

Lodwig, E. M., Leonard, M., Marroqui, S., Wheeler, T. R., Findlay, K., Downie, J. A., and Poole, P. S. 2005. Role of polyhydroxybutyrate and glycogen as carbon storage compounds in pea and bean bacteroids. Mol. Plant-Microbe Interact. 18:67-74.

Madsen, E. B., Madsen, L. H., Radutoiu, S., Olbryt, M., Rakwalska, M., Szczyglowski, K., Sato, S., Kaneko, T., Tabata, S., Sandal, N., and Stougaard, J. 2003. A receptor kinase gene of the LysM type is involved in legume perception of rhizobial signals. Nature 425:637-640.

Mitra, R. M., Gleason, C. A., Edwards, A., Hadfield, J., Downie, J. A., Oldroyd, G. E. D., and Long, S. R. 2004. A Ca ${ }^{2+} /$ calmodulin-dependent protein kinase required for symbiotic nodule development: gene identification by transcript-based cloning. Proc. Natl. Acad. Sci. U.S.A. 101:4701-4705.

Oldroyd, G. E. D., and Downie, J. A. 2004. Calcium, kinases and nodulation signalling in legumes. Nature Rev. Mol. Cell Biol. 5:566-576.

Peiter, E., Sun, J., Heckmann, A. B., Venkateshwaran, M., Riley, B. K., Otegui, M. J., Edwards, A., Freshour, G., Hahn, M. G., Cook, D. R., Sanders, D., Oldroyd, G. E. D. Downie, J. A., and Ané, J.-M. The Medicago truncatula DMI1 protein modulates cytosolic calcium signaling. Plant Physiol. In press.

Quandt, H. J., Puhler, A., and Broer, I. 1993. Transgenic root-nodules of Vicia hirsuta - a fast and efficient system for the study of gene expression in indeterminate-type nodules. Mol. Plant-Microbe Interact. 6:699706.

Radutoiu, S., Madsen, L. H., Madsen, E. B., Felle, H. H., Umehara, Y., Gronlund, M., Sato, S., Nakamura, Y., Tabata, S., Sandal, N., and Stougaard, J. 2003. Plant recognition of symbiotic bacteria requires two LysM receptor-like kinases. Nature 425:585-592.

Ramos, J., and Bisseling, T. 2003. A method for the isolation of root hairs from the model legume Medicago truncatula. J. Exp. Bot. 54:22452250 .

Riely, B. K., Lougnon, G., Ane, J. M., and Cook, D. R. 2007. The symbiotic ion channel homolog DMI1 is localized in the nuclear membrane of Medicago truncatula roots. Plant J. 149:208-216.

Saito, K., Yoshikawa, M., Yano, K., Miwa, H., Uchida, H. Asamizu, E., Sato, S., Tabata, S., Imaizumi-Anraku, H., Umehara, Y., Kouchi, H., Murooka, Y., Szczyglowski, K., Downie, J. A., Parniske, M., Hayashi, M., and Kawaguchi, M. 2007. NUCLEOPORIN85 is required for calcium spiking, fungal and bacterial symbioses, and seed production in Lotus japonicus. Plant Cell 19:610-624.

Schneider, A., Walker, S. A., Poyser, S., Sagan, M., Ellis, T. H. N., and Downie, J. A. 1999. Genetic mapping and functional analysis of a nodulation-defective mutant (sym19) of pea (Pisum sativum L.). Mol. Gen. Genet. 262:1-11.

Somasegaran, P., and Hoben, H. J. 1994. Handbook for Rhizobia. 
Springer. New York.

Stracke, S., Kistner, C., Yoshida, S., Mulder, L., Sato, S., Kaneko, T., Tabata, S., Sandal, N., Stougaard, J., Szczyglowski, K., and Parniske, M. 2002. A plant receptor-like kinase required for both bacterial and fungal symbiosis. Nature 417:959-962.

Wais, R. J., Galera, C., Oldroyd, G., Catoira, R., Penmetsa, R. V., Cook, D., Gough, C., Denarie, J., and Long, S. R. 2000. Genetic analysis of calcium spiking responses in nodulation mutants of Medicago truncatula. Proc. Natl. Acad. Sci. U.S.A. 97:13407-13412.

Walker, S. A., and Downie, J. A. 2000. Entry of Rhizobium leguminosarum bv. viciae into root hairs requires minimal nod factor specificity, but subsequent infection thread growth requires nodO or nodE. Mol. PlantMicrobe Interact. 13:754-762.

Walker, S. A., Viprey, V., and Downie, J. A. 2000. Dissection of nodulation signaling using pea mutants defective for calcium spiking induced by Nod factors and chitin oligomers. Proc. Natl. Acad. Sci. U.S.A. 97:13413-13418.

White, P. J. 2000. Calcium channels in higher plants. Biochim. Biophys. Acta 1465:171-189.
Zhu, H., Riely, B. K., Burns, N. J., and Ane, J. M. 2006. Tracing non-legume orthologs of legume genes required for nodulation and arbuscular mycorrhizal symbioses. Genetics 172:2491-2499.

\section{AUTHOR-RECOMMENDED INTERNET RESOURCES}

Accelrys website: www.accelrys.com

Center for Biological Sequence Analysis TargetP 1.1 server: www.cbs.dtu.dk/services/TargetP

EMBL-European Bioinformatics Institute CLUSTAL W webpage: www.ebi.ac.uk/Tools/clustalw/

ExPASy Proteomics tools PSORT and Tmpred: www.expasy.org/tools

National Center for Biotechnology Information BLAST website: www.ncbi.nlm.nih.gov/BLAST

Protein Homology/analogY Recognition Engine (PHYRE) website: www.sbg.bio.ic.ac.uk/phyre

University of Camridge Department of Biochemistry FUGUE v2.0: www-cryst.bioc.cam.ac.uk/ fugue 